\title{
Clinical treatment of traumatic optic neuropathy in children: Summary of 29 cases
}

\author{
MIN CHEN ${ }^{1-3}$, YAN JIANG ${ }^{1-3}$, JISHENG ZHANG $^{1,2}$ and NA LI ${ }^{1-3}$ \\ ${ }^{1}$ Department of Otorhinolaryngology Head and Neck Surgery, ${ }^{2}$ Shandong Key Laboratory of Otolaryngology \\ Head and Neck surgery and ${ }^{3}$ Shandong Provincial Key Laboratory of Digital Medicine and Computer-assisted Surgery, \\ The Affiliated Hospital of Qingdao University, Qingdao, Shandong 266003, P.R. China
}

Received December 19, 2017; Accepted July 31, 2018

DOI: $10.3892 /$ etm.2018.6637

\begin{abstract}
Clinical features and treatments for traumatic optic neuropathy (TON) in children are reported. Twenty-nine children were enrolled in the Affiliated Hospital of Qingdao University from April 1999 to May 2015 for retrospective analysis. Of these 29 patients, 5 received drug therapy, and 24 received drug therapy combined with surgical therapy. Among the patients who received surgical therapy, 23 received nasal endoscopic optic decompression under general anaesthesia, and 1 received nasal endoscopic orbital decompression under general anaesthesia. All the patients were followed up for at least 6 months. In 29 cases, 48.28\% (14/29) had visual improvement. Of the 5 patients who received drug therapy, 3 showed improvement (60\%). Of the 24 cases who received drug and surgical therapy, 11 showed improvement (45.83\%). Of the 22 patients who lost visual sensitivity, 10 showed improvement (45.45\%). Of the 7 cases with visual acuity above basic light sensitivity, 4 showed improvement (57.14\%). In conclusion, TON in children can lead to poor diagnosis and prognosis because of the difficulty of examining children and their limited language expression ability. Early ophthalmologic examination is therefore essential for children with craniofacial injuries, and proper treatment should be undertaken as soon as possible.
\end{abstract}

\section{Introduction}

Traumatic optic neuropathy (TON) is an acute injury of the optic nerve secondary to trauma; clinically, it is characterized by vision loss, dyschromatopsia, visual field defects and a relative afferent pupil defect (1). The causes of TON are thought to be multifactorial. Generally, TON can be classified as direct or indirect according to the mode of injury. Direct

Correspondence to: Dr Na Li, Department of Otorhinolaryngology Head and Neck Surgery, The Affiliated Hospital of Qingdao University, 16 Jiangsu Road, Qingdao, Shandong 266003, P.R. China E-mail: wln19831220@163.com

Key words: traumatic optic neuropathy, children
TON results from anatomical disruption of the optic nerve, whereas indirect TON results from trauma to the head or face in which the energy from the force of impact is transmitted to the bony structures that convey the optic nerve (2). Regardless of whether the cause is direct or indirect, TON remains an important cause of vision loss following traumatic head injury.

Although various management strategies have been investigated, including observation, corticosteroid treatment, surgical decompression of the optic nerve and combinations of steroids and surgical decompression $(3,4)$, little consensus has been reached among neuro-ophthalmologists regarding the appropriate treatment for TON. In addition, few studies have been published regarding the treatment of TON in children. As children have limited language expression ability, visual loss is not easily identified at an early stage, and visual acuity may not be examined when a child has a head injury. The prognosis of TON is poor, and children's lives and studies are seriously affected. Considering this, we will summarize our experience with treating TON in children based on 29 cases treated from April 1999 to May 2015 at the Affiliated Hospital of Qingdao University (Qingdao, China). We hope that our study will provide a valuable reference for the clinical treatment and prevention of TON in children.

\section{Patients and methods}

Patient enrolment. This study was approved by the Ethics Committee of the Affiliated Hospital of Qingdao University. Informed consents were freely given by the parents of the children before the study and the parents of the patients provided consent for the publication of their images.

The clinical history data of 29 children diagnosed with TON and hospitalized from April 1999 to May 2015 in our hospital were retrieved for this study. The inclusion criteria included clinical findings of visual loss in children due to head or mid-facial trauma or the presence of an ipsilateral afferent pupillary defect with normal eyesight in the other eye. Patients were excluded if their post-traumatic visual loss was not related to optic nerve dysfunction. All the children underwent a baseline neuro-ophthalmologic examination as soon as their mental state and physical condition allowed an examination of their visual function. A high-resolution computed tomography (CT) scan was conducted to evaluate the orbit and optic canal (Fig. 1). 
Surgical techniques and post-operative treatments. All patients received high-dose glucocorticoids [GEM-P (Pfizer Manufacturing Belgium NV, Belgium), 5-10 mg/kg/day] combined with vasodilators, neurotrophic agents and nutritional support after admission; dehydrators were applied in some cases. Among all the patients, 5 received drug therapy, 24 received drug combined with surgical therapy. Among the patients who received surgical therapy, 23 received nasal endoscopic optic decompression, and 1 patient received nasal endoscopic orbital decompression.

The optic canal, sphenoid sinus and skull base were examined with layer-targeted scanning CT before surgery. Routine peripheral blood, chest and electrocardiogram examinations were also conducted. The operations were performed using a tracheal cannula under general anaesthesia and well-controlled reduced blood pressure. The Messerklinger operation was adopted. Residual blood and bone chips were cleared away. After the suprasphenoid cells or the upper sphenoidalis sinus were exposed, the orbital apex was located along the orbital wall, and the surface mucosa above the optic alprotrusion was dissected to expose the canal. After that, the canal wall was thinned with a diamond drill, bone chips were removed with hooks and curettes, and the optic canal was dissected to further expose the optic nerve anterior to the common tendinous ring. Then, the hydrocele and common tendinous rings were cut along the optic nerve, and the surface of the optic nerve was washed with $1 \mathrm{ml}$ dexamethasone solution $(0.5 \%)$. The optic nerve was covered, and the sphenoid sinus and posterior ethmoid sinus were filled with gelatin sponge soaked in dexamethasone solution $(0.5 \%)$. The nasal cavity was filled with sponge or haemostatic dressing in cases of obvious bleeding. The filling in the nasal cavity was removed gradually 24-48 h after the operation. Routine antibiotics were applied to prevent infection. Glucocorticoids and neurotrophic agents were continuously applied. Post-surgical follow-up was routinely performed with a nasal endoscope.

Visual evaluation. Visual acuity and the pupillary response were evaluated daily over the course of treatment and at the end of treatment. After discharge from hospital, the patients were regularly followed up for at least 6 months and submitted to tests of visual acuity. The last visual acuity result recorded was considered the final outcome. A patient's vision was considered to have improved if the patient presented an increase of one line or more on the Snellen visual chart or demonstrated an improvement from no light perception to light perception, from light perception to hand motion, or from hand motion to finger counting.

Factors evaluated at follow-up. The follow-up factors evaluated in this study included post-treatment visual acuity (with or without light perception, hand movement, finger counting, visual acuity chart), position of the optic canal (sphenoid sinus, ethmoid sinus, poor sphenoidal sinus gasification), the interval between the time of the injury and the time of the operation, optic canal fracture, optic nerve sheath incision, and haemorrhage within the ethmoid and/or sphenoid sinus. Given that immediate or secondary visual loss was not determined in the majority of patients because they had lost consciousness or exhibited severe eyelid swelling, immediate and secondary
Table I. Clinical features of patients.

\begin{tabular}{|c|c|c|}
\hline Parameters & $\begin{array}{c}\text { No. of } \\
\text { patients }\end{array}$ & $\begin{array}{c}\text { Percentage } \\
(\%)\end{array}$ \\
\hline \multicolumn{3}{|l|}{ Sex } \\
\hline Male & 23 & 79.31 \\
\hline Female & 6 & 20.69 \\
\hline \multicolumn{3}{|l|}{ Injured side } \\
\hline Left & 18 & 62.07 \\
\hline Right & 11 & 37.93 \\
\hline Bilateral & 0 & \\
\hline \multicolumn{3}{|l|}{ Cause } \\
\hline Car accident & 9 & 31.03 \\
\hline Motorbike accident & 10 & 34.48 \\
\hline Bicycle & 1 & 3.45 \\
\hline Fall & 6 & 20.69 \\
\hline Slip & 1 & 3.45 \\
\hline Fishing rod & 1 & 3.45 \\
\hline Telegraph pole & 1 & 3.45 \\
\hline \multicolumn{3}{|l|}{ Region } \\
\hline City & 3 & 10.34 \\
\hline County & 1 & 3.45 \\
\hline Rural & 1 & 3.45 \\
\hline Countryside & 24 & 82.76 \\
\hline \multicolumn{3}{|l|}{ Complications } \\
\hline Coma & 15 & 51.72 \\
\hline Cerebrospinalrhinorrhoea & 4 & 13.79 \\
\hline \multicolumn{3}{|l|}{ Pre-treatment visual acuity } \\
\hline No light perception & 22 & 75.86 \\
\hline Light perception & 3 & 10.34 \\
\hline Hand movement & 1 & 3.45 \\
\hline Finger counting & 2 & 6.9 \\
\hline Visual acuity chart & 1 & 3.45 \\
\hline
\end{tabular}

visual loss were not evaluated in the study. Likewise, visual field and visual evoked potential data were incomplete and therefore they were not assessed.

\section{Results}

The baseline characteristics of the 29 children with TON are given in Table I. There were 29 children, aged from 4 to 17 years; 6 were between 4 and 10 years, and the remaining 23 cases were between 11 and 17 years. The duration of trauma ranged from $15 \mathrm{~h}$ to 20 days; 16 cases had a duration between $15 \mathrm{~h}$ and 3 days, while 13 cases had a duration longer than 3 days. Twenty-two patients manifested with Marcus-Gunn pupils, and 7 cases had indirect but impaired direct light responses. Contusions around the orbital soft tissues but no eye ball injury was evident in the subjects. Flash visual evoked potentials (FVEPs) were measured in 13 patients. Fracture of the optic canal was present on CT in 15 patients. Regarding other complications, 15 patients suffered from coma; 4 had cerebrospinal rhinorrhoea; 11 had abasal fracture; 15 presented sinus 
Table II. Post-treatment visual acuity and position of the optic canal.

\begin{tabular}{lcc}
\hline Parameters & $\begin{array}{c}\text { No. of } \\
\text { patients }\end{array}$ & $\begin{array}{c}\text { Percentage } \\
(\%)\end{array}$ \\
\hline $\begin{array}{l}\text { Post-treatment visual acuity } \\
\text { No light perception }\end{array}$ & 12 & 41.38 \\
Light perception & 6 & 20.69 \\
Hand movement & 1 & 3.45 \\
Finger counting & 1 & 3.45 \\
Visual acuity chart & 9 & 31.03 \\
Position of the optic canal & & \\
Sphenoid sinus & 20 & 68.96 \\
Ethmoid sinus & 7 & 24.14 \\
Poor sphenoidal sinus & 2 & 6.9 \\
gasification & & \\
\hline
\end{tabular}

bleeding (Fig. 1B); 3 had malar bone fracture; 19 presented orbital fracture (Fig. 1A); 4 presented epidural haematomas; 2 presented subdural haematomas; 2 had subarachnoid haemorrhage; 1 had facial paralysis; 3 had temporal bone fracture; 1 presented metacarpal and phalanx fractures; 3 had brain contusions and lacerations; 1 had clavicular fracture; 1 had anasal bone fracture; 2 presented oculomotor paresis; 1 had lacrimal gland contusion; 1 presented impaired dentition and mouth-opening; 1 had femur, tibia and fibula fractures; and 1 had lost some teeth.

During follow-up periods lasting from 6 months to 5 years after surgery, all the lesions in each child were epithelized, and no complications were observed. Of the 29 children, 14 showed improved visual acuity to different extents (effectiveness rate, $48.28 \%$ ): of the 5 patients who received drug therapy, 3 showed improvement (60\%). Of the 24 cases who received drug and surgical therapy, 11 showed improvement (45.83\%). Of 22 children with no light perception, 10 showed improved acuity (effectiveness rate, $45.45 \%$ ); of 7 children with acuity above light perception, 4 showed improved acuity (effectiveness rate, 57.14\%). Among the 29 patients, post-treatment acuity was equivalent to light perception in 6 patients, hand movement in 1 patient, finger counting in 1 patient, visual acuity chart in 9 patients (Table II).

\section{Discussion}

TON is primarily caused by indirect injury (5), during which external forces impact the optic canal through the displacement of the craniofacial skeleton and/or the elastic deformation of the sphenoid bone and compress and injure the optic nerve, thus leading to secondary defects in the nerve blood circulation and axonal transportation, retrospective degeneration and apoptosis of retinal ganglion cells (RGCs) and finally, visual dysfunction (6-8). The inability of children to self-assess and report symptoms after a traumatic injury can lead to the misdiagnosis of traumatic neuropathy and a poor prognosis, and early diagnosis and proper treatments are keys to a better prognosis. Thus, early ophthalmologic examinations should be required
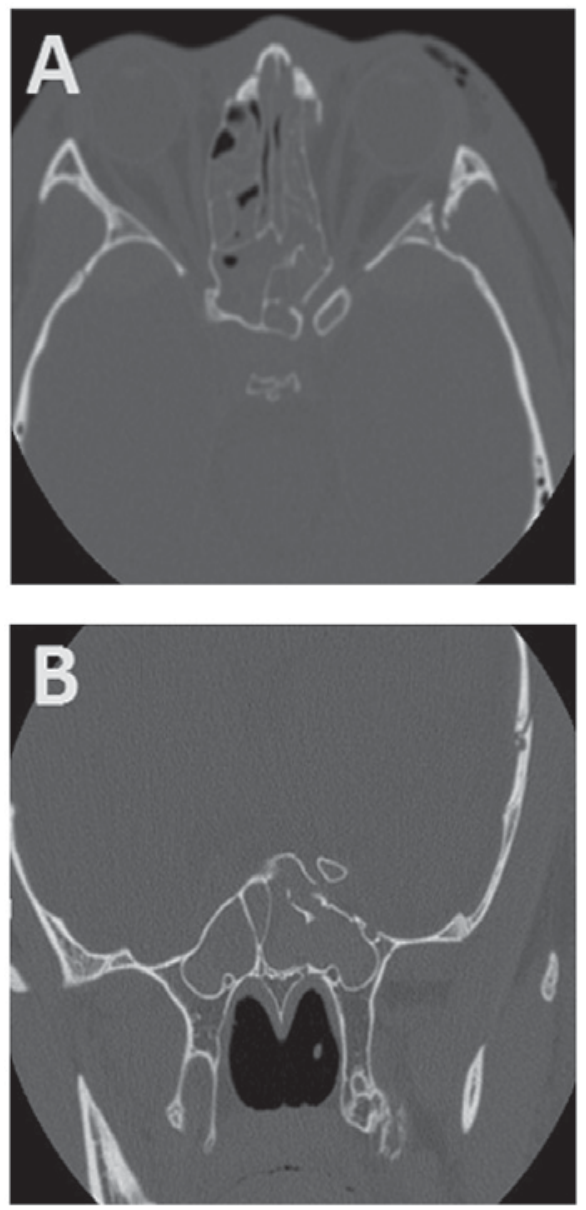

Figure 1. Typical (A) horizontal and (B) sagittal sections of optic canal CT in a 15-year-old subject. Left TON, orbital fracture and hyphaemia in the sphenoid sinus are evident. CT, computed tomography; TON, traumatic optic neuropathy.

for children with head and face injuries. In the current study, we reported 29 cases of children with TON in our hospital and summarized the related treatments and prognosis, which have rarely been reported in previous studies; thus, our retrospective study may provide clinical experience for the treatment of children with TON caused by related craniofacial injuries.

In our retrospective study, $79.31 \%$ of our subjects were boys. The $68.96 \%$ of the traumatic injuries were the result of traffic accidents, including bicycles, motorcycles, electric vehicles and cars; $20.69 \%$ were caused by falls from heights; and the remaining injuries were caused by other factors, such as falls due to slipping, injury by a fishing rod and being knocked against a wire post. Such distributions of sex and cause were consistent with previous studies (9-11). Ford et al (10) reported 26 children with TON in England, $81 \%$ of whom were boys, and the main causes of injuries were falling, traffic accidents and sport-related injuries. Gupta et al (11) reported 31 children with TON; $68 \%$ were boys, and falls from heights were the main cause. Gender differences may result from the fact that boys are more active than girls and are thus subject to a higher incidence of traumatic injuries. Furthermore, the high incidence of children from rural and countryside regions versus cities ( 86.21 vs. $13.79 \%$ ) may be accounted for by the fact that motorbikes and electric bikes are routine sources of transportation for teenagers in non-city regions, and such modes of 
transportation offer less protection than others. Additionally, children have weak safety awareness; they play in high places and can suffer fall injuries as a result.

Most children with TON also present brain injury, and some are comatose; because the main treatment after injury is for brain injury, visual loss is easily ignored. Eyelid swelling and skin injury around the eyebrow caused by orbital trauma may delay vision examinations; furthermore, because children's language abilities are poor, they cannot state their concerns, and visual loss may not be found early. The authors believe that vision should be examined in children with craniofacial trauma to allow patients with TON to be diagnosed and receive treatment as soon as possible.

Currently, the treatment of TON is controversial, and there are no evidence-based guidelines (12); treatment is still under exploration and includes mainly observation and pharmacological and surgical treatments. Routine treatment involves high-dose glucocorticoid pulse therapy combined with circulation improvement, neurotropic reagents and dehydration. Recently, erythropoietin and mesenchymal stem cell-based therapies have emerged as new treatments based on lowering tissue oedema, relieving optic nerve compression, preventing post-injury blood vessel spasm and improving axonal transduction (13-15). For the treatment of this disease, we suggest personalized treatment. All of our cases received drug therapy, 3 cases whose visual acuity improved did not receive operation, 2 cases refused operation for personal reasons, 24 cases whose visual acuity did not change after drug therapy received surgical treatment. In terms of surgical treatments, optic canal decompression can directly clean bone chips and haematomas, relieve optic nerve compression due to ischaemia, and decrease or minimize secondary nerve injuries, thus providing direct treatment (16). Consequently, we performed surgical treatment for most of the patients. This choice is theoretically supported by one relevant previous study: Gupta et al (11) recommend performing surgery as soon as possible for children with TON and believe that nasal endoscopic optic nerve decompression has many advantages, such as less trauma, clear vision and fewer complications.

Regarding the timing of surgical treatment, 24-72 h after glucocorticoid pulse therapy has been recommended as the optimal time for surgery (17). At present, there is no uniform diagnostic or treatment standard; consequently, the duration of the injury time and preoperative treatment of the patients in our study differed, and most of the surgeries were performed 3 days after injury. However, we suggest performing optic canal decompression as early as possible. Nonetheless, 1 child underwent surgery on the 21st day after injury and experienced a visual acuity improvement from 0.04 to 0.1 , and another child showed a visual acuity improvement from hand movement to 0.5 with only pharmacological treatment. Thus, even if the optimal surgical window is missed, efforts should be made to optimize the prognosis, and assessment and treatment should be individualized for different patients.

When performing surgery in children, it is difficult to distinguish the optic canal and the internal carotid artery because the sinus anatomy is different in children than in adults; sometimes the bone wall of optic nerve is thicker, so the thermal damage caused by diamond drill can not be ignored. Furthermore, the surgery space is limited because the nasal cavity and sinus are relatively small and poorly gasificated. Consequently, the relevant structures should be carefully observed with targeted CT before surgery to explore the position of the optic canal, the presence of optic nerve protrusion and the position of the optic nerve, which should be further confirmed by pressing the eyeball and observing the relative motility of the presumed optic nerve. The distance, relative position and fracture should also be noted. To minimize secondary injury to the optic canal, fine manipulation was conducted; additionally, during bone thinning with a diamond drill, the area should be washed with chilled saline, and the bones cannot be peeled until they are sufficiently thinned. Sponges soaked in saline rather than NE should be applied in cases of para-optic canal bleeding to prevent convulsion of the retinal artery and further impairment of visual acuity. As branches of the retinal artery are mainly distributed in the ventral or ventrolateral side of the optic nerve, the hydrocele should be dissected from the optic nerve above to minimize the artery injury (18).

The prognosis of TON in children is relatively poor and may affect their lives during both childhood and adulthood. As traumatic injury is the main cause of TON, it is important to minimize accidents by reinforcing safety education, controlling traffic and forbidding children from driving without supervision. In the meantime, children should wear safety gear when they are passengers in vehicles. In cases of accidents and craniofacial injury, visual function examinations should be performed for early diagnosis, and TON should be treated to improve the prognosis.

\section{Acknowledgements}

Not applicable.

\section{Funding}

This study has been funded with the 'National Natural Science Foundation of China: Regulation mechanism of histone demethylation enzyme Jarid1b on repair and regeneration of optic nerve injury (NSFC81770978)'.

\section{Availability of data and materials}

The datasets used during the present study are available from the corresponding author upon reasonable request.

\section{Authors' contributions}

NL performed the surgery, designed and drafted the study. MC collected the clinical data, analyzed the data and drafted the study. YJ collected the clinical data, analyzed the data and assisted in the modification of the manuscript. JZ helped with the data analysis. All authors agreed to be accountable for the content of the work. All authors read and approved the final manuscript.

\section{Ethics approval and consent to participate}

This study was approved by the Ethics Committee of the Affiliated Hospital of Qingdao University (Qingdao, China). 
Informed consents were freely given by the parents of the children before the study, and the parents of the patients provided consent for the publication of their images.

\section{Patient consent for publication}

Not applicable.

\section{Competing interests}

The authors declare that they have no competing interests.

\section{References}

1. al-Qurainy IA, Stassen LF, Dutton GN, Moos KF and el-Attar A: The characteristics of midfacial fractures and the association with ocular injury: A prospective study. Br J Oral Maxillofac Surg 29: 291-301, 1991.

2. Landers MB III, Bradbury MJ and Sydnor CF: Retinal vascular changes in retrograde optic atrophy. Am J Ophthalmol 86: 177-181, 1978 .

3. McClenaghan FC, Ezra DG and Holmes SB: Mechanisms and management of vision loss following orbital and facial trauma Curr Opin Ophthalmol 22: 426-431, 2011.

4. Nau HE, Gerhard L, Foerster M, Nahser HC, Reinhardt V and Joka T: Optic nerve trauma: Clinical, electrophysiological and histological remarks. Acta Neurochir (Wien) 89: 16-27, 1987.

5. Kountakis SE, Maillard AA, El-Harazi SM, Longhini L and Urso RG: Endoscopic optic nerve decompression for traumatic blindness. Otolaryngol Head Neck Surg 123: 34-37, 2000

6. Cirovic S, Bhola RM, Hose DR, Howard IC, Lawford PV, Marr JE and Parsons MA: Computer modelling study of the mechanism of optic nerve injury in blunt trauma. Br J Ophthalmol 90: 778-783, 2006

7. Steinsapir KD and Goldberg RA: Traumatic optic neuropathy: An evolving understanding. Am J Ophthalmol 151: 928-933.e2, 2011.
8. Yu-Wai-Man P: Traumatic optic neuropathy - clinical features and management issues. Taiwan J Ophthalmol 5: 3-8, 2015.

9. Goldenberg-Cohen N, Miller NR and Repka MX: Traumatic optic neuropathy in children and adolescents. J AAPOS 8: 20-27, 2004.

10. Ford RL, Lee V, Xing W and Bunce C: A 2-year prospective surveillance of pediatric traumatic optic neuropathy in the United Kingdom. J AAPOS 16: 413-417, 2012.

11. Gupta AK, Gupta AK, Gupta A and Malhotra SK: Traumatic optic neuropathy in pediatric population: Early intervention or delayed intervention? Int J Pediatr Otorhinolaryngol 71: 559-562, 2007.

12. Andersen MR and Gade E: Traumatic optic neuropathy after fall with a bamboo stick. Ugeskr Laeger 176: V05130330, 2014 (In Danish)

13. Entezari M, Esmaeili M and Yaseri M: A pilot study of the effect of intravenous erythropoetin on improvement of visual function in patients with recent indirect traumatic optic neuropathy. Graefes Arch Clin Exp Ophthalmol 252: 1309-1313, 2014.

14. Connick P, Kolappan M, Crawley C, Webber DJ, Patani R, Michell AW, Du MQ, Luan SL, Altmann DR, Thompson AJ, et al: Autologous mesenchymal stem cells for the treatment of secondary progressive multiple sclerosis: An open-label phase 2a proof-of-concept study. Lancet Neurol 11: 150-156, 2012.

15. Yu-Wai-Man P and Griffiths PG: Steroids for traumatic optic neuropathy. Cochrane Database Syst Rev 6: CD006032, 2013.

16. Vaitheeswaran K, Kaur P and Garg S: Minimal invasive transcaruncular optic canal decompression for traumatic optic neuropathy. Orbit 33: 456-458, 2014.

17. Spoor TC, Hartel WC, Lensink DB and Wilkinson MJ: Treatment of traumatic optic neuropathy with corticosteroids - correction. Am J Ophthalmol 111: 526, 1991.

18. Sandu K, Monnier P and Pasche P: Anatomical landmarks for transnasal endoscopic skull base surgery. Eur Arch Otorhinolaryngol 269: 171-178, 2012.

This work is licensed under a Creative Commons Attribution-NonCommercial-NoDerivatives 4.0 International (CC BY-NC-ND 4.0) License. 